F-18-Fluorodeoxyglucose Positron Emission

Tomography-Guided Sampling of Mediastinal Lymph Nodes in the Diagnosis of Cardiac Sarcoidosis

\title{
Simonen, Piia
}

2015-11-15

Simonen, P , Lehtonen, J , Kandolin , R, Schildt , J , Marjasuo , S , Miettinen , H , Airaksinen, J , Vihinen, T , Tuohinen , S , Haataja , P \& Kupari , M 2015 , '

F-18-Fluorodeoxyglucose Positron Emission Tomography-Guided Sampling of Mediastinal Lymph Nodes in the Diagnosis of Cardiac Sarcoidosis ' , American Journal of Cardiology , vol. 116 , no. 10 , pp. 1581-1585 . https://doi.org/10.1016/j.amjcard.2015.08.025

http://hdl.handle.net/10138/159067

https://doi.org/10.1016/j.amjcard.2015.08.025

publishedVersion

Downloaded from Helda, University of Helsinki institutional repository.

This is an electronic reprint of the original article.

This reprint may differ from the original in pagination and typographic detail.

Please cite the original version. 


\title{
F-18-Fluorodeoxyglucose Positron Emission Tomography-Guided Sampling of Mediastinal Lymph Nodes in the Diagnosis of Cardiac Sarcoidosis
}

\author{
Piia Simonen, MD ${ }^{\mathrm{a}, *}$, Jukka Lehtonen, MD ${ }^{\mathrm{a}}$, Riina Kandolin, MD ${ }^{\mathrm{a}}$, Jukka Schildt, MD ${ }^{\mathrm{b}}$, \\ Suvi Marjasuo, $\mathrm{MD}^{\mathrm{b}}$, Heikki Miettinen, $\mathrm{MD}^{\mathrm{c}}$, Juhani Airaksinen, MD ${ }^{\mathrm{d}}$, Tapani Vihinen, $\mathrm{MD}^{\mathrm{d}}$, \\ Suvi Tuohinen, $\mathrm{MD}^{\mathrm{e}}$, Petri Haataja, $\mathrm{MD}^{\mathrm{e}}$, and Markku Kupari, MD ${ }^{\mathrm{a}}$
}

\begin{abstract}
Histologic proof of granulomatous inflammation is prerequisite for the diagnosis of cardiac sarcoidosis (CS). Because of the limited sensitivity of endomyocardial biopsy (EMB), confirmation of sarcoidosis often has to be acquired from extracardiac biopsies. We set out to review our experience of F-18-fluorodeoxyglucose positron emission tomography (F-18-FDG PET) in guiding extracardiac tissue biopsies in suspected CS. We included in this work 68 consecutive patients with proved CS who had undergone cardiac F-18-FDG PET with $(n=57)$ or without whole-body imaging as part of initial diagnostic evaluation. Their hospital charts, imaging studies, and diagnostic biopsies were reviewed in retrospect. Whole-body PET images showed extracardiac foci of abnormally high F-18-FDG uptake in 39 of 57 patients, of whom 38 had involvement of mediastinal lymph nodes (MLN). Parallel F-18-FDG uptake was found in other lymph nodes $(n=10)$, lungs $(n=9)$, liver $(n=3)$, spleen $(n=2)$, and thyroid gland $(n=1)$. Adding the mediastinal findings at cardiac PET without whole-body imaging, abnormal F-18-FDG uptake in MLN was found in totally 43 of the 68 patients with CS (63\%). Histology of systemic sarcoidosis was known at presentation of cardiac symptoms in 8 patients. Of the 60 patients with missing histology, 24 patients underwent mediastinoscopy for sampling of PET-positive MLN, most often $(n=20)$ after nondiagnostic EMB; microscopy revealed diagnostic noncaseating granulomatous inflammation in 24 of the 24 cases (sensitivity 100\%). In the remaining 36 patients, sarcoidosis histology was confirmed by EMB $(n=30)$, by biopsy of lungs $(n=2)$ or peripheral lymph nodes $(n=2)$, or at autopsy $(n=1)$ or posttransplantation $(n=1)$. In conclusion, MLN accumulate F-18-FDG at PET in most patients with CS and provide a highly productive source for diagnostic biopsies either primarily or subsequent to nondiagnostic EMB. (c) 2015 Elsevier Inc. All rights reserved. (Am J Cardiol 2015;116:1581-1585)
\end{abstract}

Cardiac sarcoidosis (CS) presents to the cardiology services as an isolated heart condition much more commonly than as one manifestation of a known or clinically evident systemic sarcoidosis. ${ }^{1,2}$ Its main forms of presentation are atrioventricular conduction block, ventricular tachyarrhythmias, and heart failure, either alone or in various combinations. ${ }^{1-3}$ Distinguishing CS from more common myocardial conditions, ischemic or nonischemic, is

${ }^{\mathrm{a}}$ Division of Cardiology, Heart and Lung Center and ${ }^{\mathrm{b}}$ Department of Nuclear Medicine, HUS Medical Imaging Center, Helsinki University Central Hospital, Helsinki, Finland; ${ }^{\circ}$ Heart Center, Kuopio University Hospital, Kuopio, Finland; ${ }^{\mathrm{d}}$ Heart Center, Turku University Hospital, Turku, Finland; and ${ }^{\mathrm{e}}$ Heart Hospital, Tampere University Hospital, Tampere, Finland. Manuscript received June 28, 2015; revised manuscript received and accepted August 18, 2015.

The study was supported by a Finnish government grant for medical research, the Finnish Foundation for Cardiovascular Research, Helsinki, Finland, and the Finnish Cultural Foundation, Helsinki, Finland.

See page 1584 for disclosure information.

*Corresponding author: Tel: $(+358)$ 9-471-72442; fax: $(+358)$ 9-47174574.

E-mail address: piia.simonen@hus.fi (P. Simonen). critical for the patient care yet frequently a tough challenge. The only absolute diagnosis of CS comes from finding typical granulomatous myocarditis in a sample of myocardium without other explanation. However, because of the patchy distribution of sarcoid granulomas, endomyocardial biopsy (EMB) more often misses than hits areas diagnostic of CS resulting in a sensitivity no better than $19 \%$ to $32 \%{ }^{4-6}$ CS can be diagnosed with less but clinically sufficient certainty also if the histology is proved in an extracardiac tissue sample and clinical manifestations and findings at cardiac gadolinium-enhanced magnetic resonance imaging (Gd-MRI) or F-18-fluorodeoxyglucose positron emission tomography (F-18-FDG PET) are compatible with myocardial involvement. ${ }^{6}$ Since the first published cardiac F-18-FDG PET images in $\mathrm{CS}^{7}$, with focally increased myocardial glucose uptake signaling inflammatory activity, PET has gained wide use in the initial diagnosis, assessment of disease activity, and monitoring of treatment response in CS. ${ }^{8-10}$ Moreover, whole-body PET can uncover hidden inflammatory foci in extracardiac organs, ${ }^{11}$ thus suggesting targets for diagnostic tissue biopsies. We set out to review our nationwide CS registry ${ }^{2}$ for details of the use of F-18-FDG PET in the detection of 


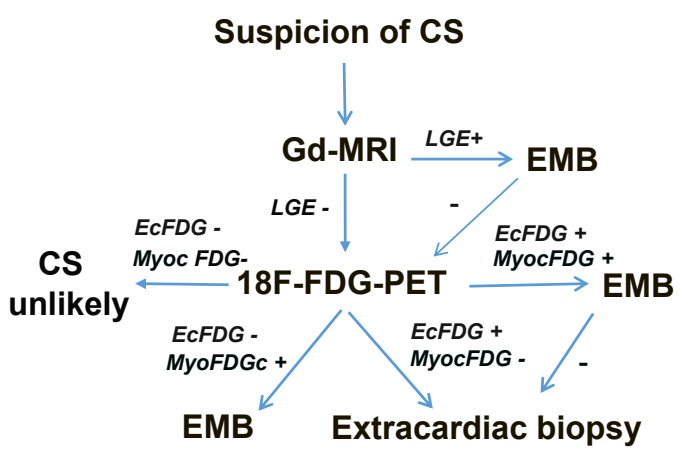

Figure 1. Summary of the diagnostic strategy in suspicion of CS during the study period. EcFDG = abnormal extracardiac F-18-FDG uptake; LGE $=$ late gadolinium enhancement; MyocFDG $=$ abnormal myocardial F-18-FDG uptake.

CS. In the present work, we focus on extracardiac PET findings and show their utility in the diagnosis of CS.

\section{Methods}

The registry of Myocardial Inflammatory Diseases in Finland has collected all patients diagnosed in our country with clinically manifest CS since the turn of 1990s. ${ }^{2}$ The criteria of CS required for inclusion in the registry have been detailed in our earlier reports. ${ }^{2,5,12}$ In brief, either myocardial or extracardiac histology of noncaseating granulomatous inflammation has been mandatory in addition to clinical manifestations and findings at cardiac imaging compatible with CS. From this database, we identified 72 patients with CS who had undergone F-18-FDG PET from 2005 to early 2013 as part of their diagnostic assessment after admission. Of them, 4 patients were excluded because of technically inadequate PET studies. The remaining 68 patients constitute the present study population. Their hospital charts were scrutinized in retrospect for demographics, cardiac signs and symptoms, laboratory tests, imaging studies, and diagnostic biopsies, and the data pertinent to the present work were collected for analysis. The study was performed according to the principles of the Declaration of Helsinki. The Ethics Committee for the Department of Medicine, Helsinki University Central Hospital, approved the study protocol, and the Myocardial Inflammatory Diseases in Finland registry study has been approved by the national ethical review board (STM/1219/2009). A proportion of the CS population ( 25 of the 68 patients) has been reported earlier.

During the period covered by our work, patients presenting with etiologically unknown high-grade atrioventricular conduction disturbance, ventricular tachyarrhythmia, or heart failure were initially examined clinically and with routine laboratory tests, chest x-rays, and echocardiography. Once ischemic heart disease had been excluded, either clinically or at coronary angiography, patients usually underwent cardiac Gd-MRI unless it was contraindicated. If MRI was suggestive of myocardial inflammation by showing Gd late enhancement with or without edema or abnormal LV wall thinning or thickening, ${ }^{13}$ a right or left ventricular EMB was done aiming at the site of myocardial abnormalities identified in MRI. Cardiac F-18-FDG PET, usually complemented with whole-body image acquisition, was done (1) if MRI was contraindicated, (2) if the results of MRI were nonrevealing but suspicion of CS persisted, or (3) if the post-MRI EMB was nondiagnostic. If PET showed focally increased F-18-FDG uptake in the myocardium, EMB was again the next procedure. Extracardiac tissue biopsy was done, provided there was F-18-FDG accumulation outside the heart if (1) there was no abnormal myocardial F-18-FDG accumulation or (2) the post-PET EMB was negative. Figure 1 illustrates this diagnostic strategy.

Most F-18-FDG PET studies (58 of 68) were done at the Department of Nuclear Medicine, Helsinki University Central Hospital. The patients fasted for at least 12 hours before the examination, and blood glucose level had to be $<7.0 \mathrm{mmol} / \mathrm{L}$ before the study. After an intravenous injection of F-18-FDG (303 $\pm 57 \mathrm{MBq})$, the uptake phase lasted at least 60 minutes. PET images were acquired with a Philips Gemini PET/computed tomographic (CT) scanner. For the whole-body images, scanning was performed from the proximal femoral region to the head. Cardiac image acquisition was done thereafter. Within 1 week from the PET/CT scan, a Tc-99m-tetrofosmin myocardial perfusion imaging at rest was performed. The details of these imaging methods have been described previously., ${ }^{5,12}$ Ten patients underwent PET in 3 other Finnish university hospitals according to their local routines. All PET studies, including the ones done elsewhere, were analyzed by an experienced nuclear medicine specialist (JS) at the core institution in Helsinki. Any pathologic uptake of F-18-FDG outside the heart was located and recorded, including the presence, number, and size of F-18-FDG-positive mediastinal lymph nodes (MLN). In cardiac PET, normal myocardial metabolism was defined as either complete suppression of F-18-FDG uptake or diffuse uptake without any areas of focally increased activity (i.e., without F-18-FDG "hot spot"). The Tc-99m-tetrofosmin myocardial perfusion at rest was compared with the F-18-FDG uptake in the 17-segment LV model, and each segment was visually classified as having either (1) normal perfusion and normal metabolism, (2) abnormal perfusion or abnormal metabolism (i.e., "hot spot"), or (3) abnormal perfusion and abnormal metabolism.

All statistical analyses were performed with the SPSS for Windows 19.0 Statistics (SPSS, Chicago, Illinois). The data are presented as mean \pm SD for continuous variables and as absolute numbers or percentages for categorical variables, unless otherwise noted. Normal distribution and homogeneity of variance were checked before further analyses. Between-group comparisons for continuous variables were made with the Student's 2-sided $t$ test. Comparisons of discrete variables between groups were assessed with the chi-square test or Fisher's exact test. All statistical tests were 2 tailed, and $\mathrm{p}<0.05$ was regarded as statistically significant.

\section{Results}

The CS population comprised 47 women and 21 men with a mean age of $50 \pm 9$ years. Their main presenting cardiac manifestations were, in order of decreasing frequency, complete atrioventricular block $(\mathrm{n}=37,54 \%)$, sustained ventricular tachycardia $(\mathrm{n}=18,26 \%)$, congestive 


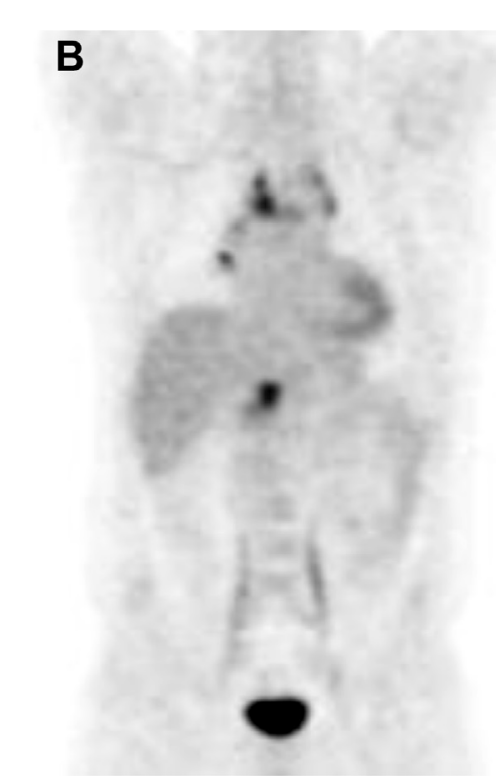

Figure 2. Examples of cardiac $(A)$ and whole-body $(B)$ F-18-FDG PET studies showing "hot" MLN together with foci of activity in the heart and outside the

thoracic cavity.

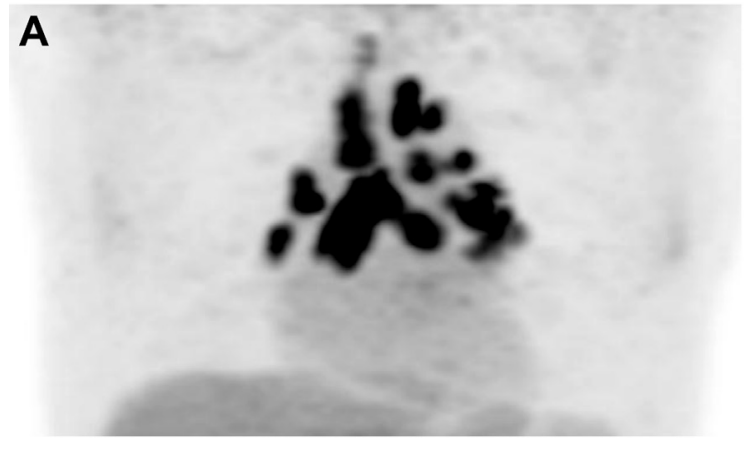

Table 1

Details of extracardiac localization of F-18-FDG uptake at whole-body PET in 57 patients with $\mathrm{CS}$

\begin{tabular}{lc}
\hline Foci of abnormal extracardiac F-18-FDG uptake & $\mathrm{n}$ \\
\hline Mediastinal lymph nodes (only) & 21 \\
Mediastinal lymph nodes, lungs & 6 \\
Mediastinal and extrathoracic lymph nodes & 6 \\
Mediastinal and extrathoracic lymph nodes, lungs & 2 \\
Mediastinal and extrahoracic lymph nodes, spleen & 1 \\
Mediastinal and extrathoracic lymph nodes, spleen and liver & 1 \\
Mediastinal lymph nodes, lungs, liver and thyroid gland & 1 \\
Liver (only) & 1 \\
No extracardiac uptake & 18
\end{tabular}

$\mathrm{CS}=$ cardiac sarcoidosis; F-18-FDG = F-18-fluorodeoxyglucose; $\mathrm{PET}=$ positron emission tomography.

heart failure $(\mathrm{n}=7,10 \%)$, ventricular fibrillation $(\mathrm{n}=4$, $6 \%)$, and multiple ventricular premature beats $(\mathrm{n}=2,3 \%)$. An impaired LV function at echocardiography on admission (ejection fraction $<50 \%$ ) was found in 33 patients $(49 \%)$, and Gd-MRI revealed pathologic LV wall late enhancement in 33 of the 43 patients studied (77\%). Cardiac PET was abnormal in 62 of 68 patients. It showed both an F-18-FDG "hot spot" and a myocardial perfusion defect in 52 patients and either a perfusion defect or a "hot spot" in 10 patients. Of the 6 patients with normal cardiac F-18-FDG PET, 5 had pathologic late enhancement at Gd-MRI.

Cardiac and whole-body PET combined showed pathologically increased F-18-FDG uptake (Figure 2) in MLN in totally 43 of the $68 \mathrm{CS}$ patients $(63 \%)$. Most of the PET-positive lymph nodes were located in the right upper and lower paratracheal regions, subcarinally and in the subaortic and para-aortic areas. Their number ranged from 2 to 14 per patient (mean 4), and their size averaged $1.2 \pm$ $0.3 \mathrm{~cm}$. Table 1 gives a complete list of sites of pathologic extracardiac F-18-FDG uptake in the 57 patients who

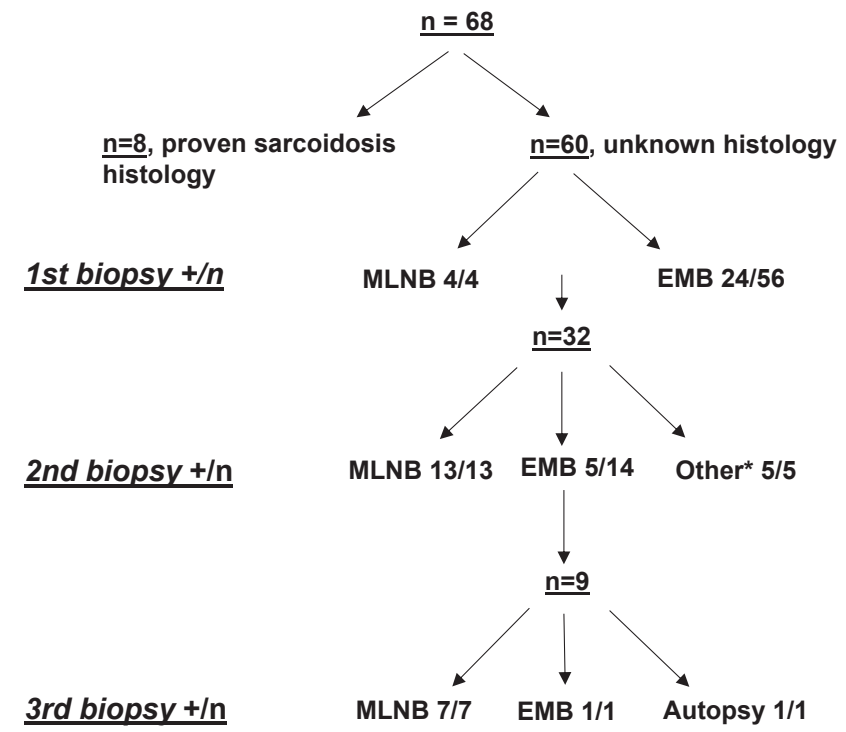

Figure 3. The path to the histologic proof of sarcoidosis. In 60 patients with unknown histology at presentation, 101 tissue samples were taken and studied histologically in 3 successive biopsy rounds. Totally 24 MLNB and 71 EMBs were done. Granulomatous inflammation was ultimately confirmed in 24 of 24 MLNBs and in 30 of 71 EMBs. "**" Indicates "other" biopsy sites that included 2 transbronchial lung biopsies, 2 inguinal lymph node biopsies, and 1 post-transplantation study of the native heart; $+/ \mathrm{n}=$ number of patients with positive biopsies per number of patients biopsied. MLNB = mediastinal lymph node biopsies.

underwent whole-body PET imaging. The data show that although 18 of 57 patients (32\%) had F-18-FDG accumulation outside mediastinum, all except 1 of them also had parallel MLN involvement. None of the 6 patients with normal cardiac PET had abnormal extracardiac F-18-FDG activity, indicating that these 6 studies (9\% of all) were completely false negative. 
Figure 3 summarizes the path of successive biopsies to the ultimate histologic diagnosis in the 60 patients with missing histology of sarcoidosis at presentation. Biopsy of MLN via mediastinoscopy was done as the first procedure in 4 patients and after failing EMBs in 20 patients. It confirmed sarcoidosis histology in 24 of 24 attempts (sensitivity $100 \%)$. Fifty-six patients underwent solitary or repeated EMBs (totally 71) that ultimately proved the histology of sarcoidosis in 30 patients (cumulative sensitivity 54\%). In the remaining 6 patients, biopsy of lung or peripheral lymph nodes confirmed sarcoidosis in 4 patients and the histologic diagnosis was made only after transplantation or at autopsy in 2 patients. One of the 24 patients who underwent mediastinoscopy had transient hoarseness of voice but no serious complications occurred. After 71 EMBs, 1 patient had a small amount of pericardial effusion that resolved spontaneously and 1 other patient showed mild new tricuspid regurgitation.

\section{Discussion}

The key finding of our observational series was that, in CS, MLN show abnormal F-18-FDG uptake at PET in most patients and that their sampling provides a highly productive means to prove the histology of sarcoidosis. Otherwise, our work showed that the sensitivity of EMB in CS is better than previously reported and can exceed $50 \%$ if the procedure is guided by findings at cardiac imaging and repeated if initially negative. This is noteworthy because, as our wholebody PET studies indicated (see Table 1), sarcoidosis is truly isolated to the heart in $1 / 3$ of CS and cannot be histologically confirmed without microscopy of myocardial samples.

Diagnosis of CS has important prognostic and therapeutic implications to the individual involved. It nearly invariably sets off long-term immunosuppressive treatment and also constitutes an indication to consider a prophylactic intracardiac cardioverter defibrillator. ${ }^{6}$ Therefore, and given the potential complications of these treatments, the diagnosis should leave no room for doubt. An absolute diagnosis of CS only comes from a sample of myocardium showing typical noncaseating granulomatous histology in the absence of other explanations. Histologically proved extracardiac sarcoidosis combined with findings at cardiac imaging indicative of nonischemic myocardial involvement constitutes a less certain yet clinically sufficient diagnosis of CS. ${ }^{6}$ Although diagnosing CS without any histologic proof has been possible, ${ }^{14}$ this policy was plainly dismissed in the recent expert consensus statement from the Heart Rhythm Society. ${ }^{6}$ The consensus report ${ }^{6}$ preferred extracardiac tissue biopsies over EMB in suspected CS with arguments of safety and yield. In our practice, EMB has hitherto been the primary procedure for histologic diagnosis. Worthy of note, we have shown both here and previously ${ }^{5}$ that targeting endomyocardial sampling, with the help of modern cardiac imaging, combined with willingness to repeat the procedure, significantly increases the yield from EMB. Electroanatomic mapping-guided myocardial sampling provides another means to improve the sensitivity of EMB.

MLN "hot" (i.e., avid for F-18-FDG) at PET served here as a perfect target for extracardiac biopsies to confirm the histology of sarcoidosis after nonrevealing EMB. Because 2/3 of our patients in need of histologic diagnosis had "hot" mediastinum at PET, starting with mediastinoscopy instead of EMB had certainly simplified and shortened the diagnostic path to a considerable extent (see Figure 3). In this light, our results clearly support the new consensus statement favoring extracardiac biopsies over EMB. ${ }^{6}$ In contrast, EMB is still needed to confirm the diagnosis of isolated CS and it also enables other than histologic studies, like myocardial gene expression profiling, that may help detect $\mathrm{CS} .^{16}$

Abnormal extracardiac uptake of F-18-FDG at PET was observed much more commonly in the mediastinum than in all other extracardiac sites together (63\% vs $26 \%$ ). Teirstein et $\mathrm{al}^{17}$ also found in their whole-body PET studies that MLN constitute by far the most common site of hidden extracardiac disease in sarcoidosis. Lymphatic drainage from the left side of the heart is collected in the right upper paratracheal nodes ${ }^{18}$ that could explain why granulomatous inflammation in CS frequently involves the mediastinal nodes but spares the hilar nodes. ${ }^{5,19}$ Although MLN provide a yielding biopsy target in suspected CS, other causes of noncaseating granulomatous lymphadenitis must always be excluded, and as Thachil et $\mathrm{al}^{20}$ have shown, particularly, cautious interpretation is needed in areas endemic for tuberculosis.

\section{Disclosures}

The authors have no conflicts of interest to disclose.

1. Okura Y, Dec GW, Hare JM, Kodama M, Berry GJ, Tazelaar HD, Bailey KR, Cooper LT. A clinical and histopathologic comparison of cardiac sarcoidosis and idiopathic giant cell myocarditis. J Am Coll Cardiol 2003:41:322-329.

2. Kandolin R, Lehtonen J, Airaksinen J, Vihinen T, Miettinen H, Ylitalo K, Kaikkonen K, Tuohinen S, Haataja P, Kerola T, Kokkonen J, Pelkonen M, Pietilä-Effati P, Utrianen S, Kupari M. Cardiac sarcoidosis: epidemiology, characteristics and outcome over 25 years in a nationwide study. Circulation 2015;131:624-632.

3. Blauwet LA, Cooper LT. Idiopathic giant cell myocarditis and cardiac sarcoidosis. Heart Fail Rev 2013;18:733-746.

4. Uemura A, Morimoto S, Hiramitsu S, Kato Y, Ito T, Hishida H. Histologic diagnostic rate of cardiac sarcoidosis: evaluation of endomyocardial biopsies. Am Heart J 1999;138:299-302.

5. Kandolin R, Lehtonen J, Graner M, Schildt J, Salmenkivi K, Kivistö SM, Kupari M. Diagnosing isolated cardiac sarcoidosis. J Intern Med 2011;270:461-468.

6. Birnie DH, Sauer WH, Bogun F, Cooper JM, Culver DA, Duvernoy CS, Judson MA, Kron J, Mehta D, Nielsen JC, Patel AR, Ohe T, Raatikainen P, Soejima K. HRS expert consensus statement on the diagnosis and management of arrhythmias associated with cardiac sarcoidosis. Heart Rhythm 2014;11:1305-1323.

7. Takeda N, Yokoyama I, Hiroi Y, Sakata M, Harada T, Nakamura F, Murakawa Y, Nagai R. Positron emission tomography predicted recovery of complete A-V nodal dysfunction in a patients with cardiac sarcoidosis. Circulation 2002;105:1144-1145.

8. Kruger S, Buck AK, Mottahgy FM, Pauls S, Schelzig H, Hombach V, Reske SN. Use of integrated FDG-PET/CT in sarcoidosis. Clin Imaging 2008:32:269-273.

9. Youssef G, Leung E, Mylonas I, Nery P, Williams K, Wisenberg G, Gulenchyn KY, deKemp RA, DaSilva J, Birnie D, Wells GA, Beanlands RS. The use of ${ }^{18} \mathrm{~F}-\mathrm{FDG}$ PET in the diagnosis of cardiac sarcoidosis: a systematic review and meta-analysis including the Ontario experience. J Nucl Med 2012;53:241-248.

10. Skali H, Schulman AR, Dorbala S. ${ }^{18}$ F-FDG PET/CT for the assessment of myocardial sarcoidosis. Curr Cardiol Rep 2013;15: $370-381$ 
11. Sobic-Saranovic D, Artiko V, Obradovic V. FDG PET imaging in sarcoidosis. Semin Nucl Med 2013;43:404-411.

12. Kandolin R, Lehtonen J, Kupari M. Cardiac sarcoidosis and giant cell myocarditis as causes of atrioventricular block in young and middleaged adults. Circ Arrhythm Electrophysiol 2011;4:303-309.

13. Youssef G, Beanlands RS, Birnie DH, Nery PB. Cardiac sarcoidosis: applications in imaging and directing treatment. Heart 2011;97: 2078-2087.

14. Jpn Ministry Health Welfare, Diagnostic standard and guidelines for sarcoidosis. Jpn J Sarcoidosis Granulomatous Disord 2007;27:89-102.

15. Nery PB, Keren A, Healey J, Leug E, Beanlands RS, Birnie DH. Isolated cardiac sarcoidosis: establishing the diagnosis with mappingguided endomyocardial biopsy. Can J Cardiol 2013;29:1015. e1-1015.e3.

16. Lassner D, Kühl U, Siegismund CS, Rohde M, Elezkurtaj S, Escher F, Tschöpe C, Gross UM, Poller W, Schultheiss HP. Improved diagnosis of giant cell myocarditis and cardiac sarcoidosis by myocardial gene expression profiling. Eur Heart J 2014;35:2186-2195.

17. Teirstein AS, Machac J, Almeida O, Lu P, Padilla ML, Iannuzzi MC, Results of 188 whole-body positron emission tomography scans in 137 patients with sarcoidosis. Chest 2007;132:1949-1953.

18. Loukas M, Abel N, Tubbs RS, Grabska J, Birungi J, Anderson RH. The cardiac lymphatic system. Clin Anat 2011;24:684-691.

19. Otsuka K, Terasaki F, Eishi Y, Shimomura H, Ogura Y, Horii T, Isomura T, Suma H, Kitaura Y. Cardiac sarcoidosis underlies idiopathic dilated cardiomyopathy: importance of mediastinal lymphadenopathy in the differential diagnosis. Circ J 2007;71:1937-1941.

20. Thachil A, Christopher J, Sastry BKS, Reddy KN, Tourani VK, Hassan A, Raju BS, Narasimhan C. Monomorphic ventricular tachycardia and mediastinal adenopathy due to granulomatous infiltration in patients with preserved ventricular function. J Am Coll Cardiol 2011;28: $48-55$. 